\title{
HIGHER-ORDER-MODE DAMPER TESTING AND INSTALLATION IN THE ADVANCED PHOTON SOURCE 352-MHZ SINGLE-CELL RF CAVITIES*
}

\author{
G. Waldschmidt, L. Morrison, D. Horan, G. Pile, N. DiMonte \\ Argonne National Laboratory \\ Argonne, IL 60439, U.S.A.
}

\begin{abstract}
Higher-order-mode dampers were recently installed in the storage ring rf cavities at the Advanced Photon Source to reduce longitudinal coupled-bunch instability. It was discovered that the $535-\mathrm{MHz}$ cavity monopole mode created beam instability at beam currents in excess of 85 $\mathrm{mA}$ causing effective horizontal emittance blowup (due to dispersion). Methods of compensating for the instability by detuning the cavities and adjusting the cavity water temperature were becoming more difficult at higher beam currents as tests were performed to prepare for eventual 300-mA beam current operation. Electric field passive dampers located on the median plane of each cavity were determined to be the most promising solution. Simulation models were created and verified with low-power testing of the dampers. High-power testing of the dampers as well as conditioning of the damper ceramic load were also performed at the APS $352-\mathrm{MHz}$ rf test stand and compared with simulation results. Preliminary test results will be discussed.
\end{abstract}

\section{INTRODUCTION}

Higher-order-mode (HOM) dampers are often installed in accelerators to sufficiently damp modes that degrade the beam quality or produce instabilities. To mitigate the effects of the HOMs at the Advanced Photon Source (APS), the physical length of each of the storage ring cavities was staggered by $0.3 \mathrm{~mm}$ during initial construction. This has reduced the collective effects of the sixteen cavities at many HOM frequencies although the benefits are reduced due to the tolerances of the cooling temperature regulation as well as the slight asymmetry of the cavity operating power levels.

The APS does not require HOM dampers in the storage ring cavities for routine accelerator operations below 100 $\mathrm{mA}$ with nominal fill patterns. However, as $300-\mathrm{mA}$ beam current is investigated, the removal of HOMs will become necessary [1]. In fact, at 100-mA, accommodations for the HOMs have required cavity detuning and temperature adjustment in order to prevent beam instability. These methods have been effective over the years, but have become more difficult to implement due to a combination of $\mathrm{rf}$ frequency evolution in the storage ring and explorations of various fill patterns.

The 535-MHz monopole HOM has been found to cause longitudinal beam instability at levels near operating conditions. It has become increasingly difficult to find

*This work is supported by the U.S. Department of Energy, Office of Basic Energy Sciences, under Contract No. W-31-109-ENG-38. stable operating points due to its dependence on gap voltage and cavity temperature. This will become much more problematic as higher beam currents are used and multiple HOMs are present. In order to evaluate the physical effects of dampers in the storage ring as well as their effects on the longitudinal impedance spectrum, four dampers were tested and installed onto four single-cell cavities.

\section{DAMPER DESIGN}

Design and manufacturing considerations for dampers in the APS storage ring have been discussed in detail $[2,3]$. Since the 535-MHz HOM has a strong electric field component along the equatorial plane of the cavity, a coaxial e-probe damper was chosen as shown in Fig. 1. Since the radial component of the $352-\mathrm{MHz}$ accelerating mode is zero along the mid-plane, no rejection filter was needed to prevent excessive deQing.

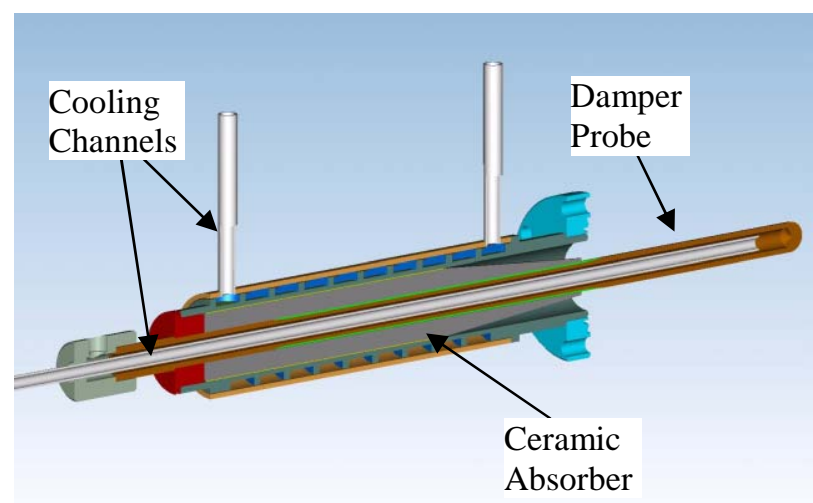

Figure 1: Mechanical design of damper.

The ceramic load is made of AlN with $40 \%$ SiC. It is compatible with ultrahigh vacuum applications and as a result does not require a separate window as a vacuum barrier. In order to dissipate kilowatts of power, it was found that a total length of eight inches was sufficient with proper water cooling. A three-inch taper was manufactured at its leading edge to minimize reflections from the propagating TEM mode. The remaining fiveinch length completely fills the volume between the probe and the outer conductor.

The center conductor is a half-inch-diameter solid piece of glidcop. It was gundrilled and fitted with a tube to allow water circulation through the probe. Since the probes were to be inserted into the cavity vacuum, 
particular effort was made to avoid a water leak. As a result, the probes were x-rayed to ensure a uniform 0.080 " wall thickness.

The coaxial damper was designed to have a characteristic impedance of $50 \mathrm{Ohms}$ although there was a step discontinuity from the cavity port. The ceramic has a relative permittivity of 47 and a loss tangent of 0.085 at $500 \mathrm{MHz}$.

\section{MODELING AND EXPERIMENT}

\section{Low-Power Testing at $535 \mathrm{MHz}$}

A low-power test setup was used with a spare singlecell storage ring cavity that was either sealed or kept in constant positive pressure with a nitrogen dewar. An HP network analyzer was used to take the measurements. Low-power testing revealed that the HOM could not be produced as anticipated along the equatorial plane with an electric field probe or in other ports elsewhere on the cavity with a magnetic field probe properly oriented.

As a result, simulation models were created to produce this mode in a low-power test setup. After the simulation models corroborated the initial setup, it was discovered that a foreign object was deQing the cavity particularly strongly at the HOM but not at the accelerating mode. Once resolved, two magnetic field probes were used for S21 measurements to evaluate the effectiveness of the dampers.

Table 1 shows the effect on the HOM due to the damper as it is inserted into the cavity at different depths. The depth is modified by adding spacers to the damper flange thereby reducing the damper penetration depth into the cavity. In the table, the probe depth is the distance the damper probe extends beyond the cavity port flange. Since the port extends for about 2.5 inches, the total insertion depth of the probe past the cavity wall is then 2.5 inches less than the probe depth. For reference, the HOM values with no damper are given in the first row of data.

Table 1: Frequency shift and deQing of the $535-\mathrm{MHz}$ HOM at various damper probe insertion depths.

\begin{tabular}{|c|c|c|c|c|}
\hline $\begin{array}{c}\text { Probe } \\
\text { Depth } \\
\text { (inches) }\end{array}$ & $\begin{array}{c}\text { Freq } \\
(\mathrm{MHz})\end{array}$ & $\begin{array}{c}\Delta \text { Freq } \\
(\mathrm{kHz})\end{array}$ & $\mathrm{Q}$ & $\begin{array}{c}\mathrm{Q}^{\mathrm{Q}} \mathrm{Q}_{0} \\
\mathrm{deQ} \text { eing }\end{array}$ \\
\hline None & 535.694 & ----- & 44200 & ----- \\
\hline 3.0 & 535.685 & 9.33 & 38200 & 1.16 \\
\hline 3.5 & 535.665 & 29.04 & 27600 & 1.60 \\
\hline 3.75 & 535.646 & 48.28 & 19900 & 2.22 \\
\hline 4.0 & 535.620 & 73.70 & 12700 & 3.48 \\
\hline 4.25 & 535.590 & 104.01 & 8400 & 5.26 \\
\hline 4.5 & 535.550 & 143.66 & 4700 & 9.34 \\
\hline
\end{tabular}

\section{High-Power Testing at $352 \mathrm{MHz}$}

When the damper was placed in the equatorial plane of the cavity, simulation models, like the one in Fig. 2, have shown that little fundamental mode power was

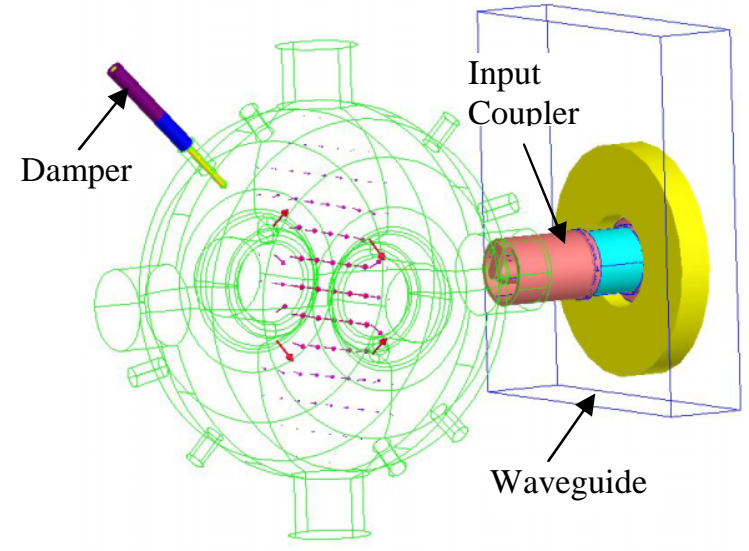

Figure 2: Simulation model for evaluating the deQing of the $\mathrm{TM}_{010}$ mode by the coaxial damper positioned on the equatorial plane of a typical storage ring cavity.

Table 2: Simulated power loss in the damper ceramic and damper probe at various insertion depths into the cavity assuming $100 \mathrm{~kW}$ of fundamental mode input power.

\begin{tabular}{|c|c|c|}
\hline $\begin{array}{c}\text { Probe Depth } \\
\text { (inches) }\end{array}$ & $\begin{array}{c}\mathrm{P}_{\text {loss }}: \text { Ceramic } \\
(\mathrm{W})\end{array}$ & $\begin{array}{c}\mathrm{P}_{\text {loss }}: \text { Probe } \\
(\mathrm{W})\end{array}$ \\
\hline 2.5 & 0.001 & 10 \\
\hline 3.0 & 0.037 & 50 \\
\hline 3.5 & 0.060 & 115 \\
\hline 4.0 & 0.180 & 206 \\
\hline 4.5 & 0.020 & 259 \\
\hline
\end{tabular}

absorbed by the damper. Table 2 shows the simulation results of the power intercepted by the damper. The damper ceramic intercepts minimal power levels while the damper probes intercept small but measurable levels, affecting the unloaded cavity $\mathrm{Q}$ by less than $0.5 \%$. Note that the ceramic power levels are small and therefore more subject to numerical noise.

High-power testing performed in the APS 352-MHz rf test stand corroborated this analysis. At low power levels, the losses in the ceramic were too small to be accurately measured using calorimetry.

\section{High-Power Conditioning at $352 \mathrm{MHz}$}

Since it was verified that little power was absorbed by the damper at its operating location in the rf test stand, there was no need to perform high-power testing on each of them prior to installation. However, before the dampers were installed in the ring, it was necessary to rate the damper ceramics for power-handling limits to determine their compatibility with high-power and highfield environments. A transmission line as well as a 535$\mathrm{MHz}$ input coupler were designed for the purpose of supplying power to the damper ceramic.

However, since the damper would operate in an ultrahigh vacuum environment, it was also necessary to condition the ceramics. Conditioning rf components such as ceramics prior to installation into the storage ring has 
substantially reduced the time needed to prepare the ring for normal operation. This has been done routinely for input coupler ceramics and tuners since the installation of the storage ring test stand at the APS.

In order to rate the damper ceramic and prepare it for an ultrahigh vacuum environment, it was decided to condition the dampers on the front cover of the single-cell cavity where the damper probe makes roughly a $30^{\circ}$ angle with the electric field of the fundamental mode. A substantial amount of power is coupled into the damper and is absorbed by the ceramic damper. With this configuration, $1 \mathrm{~kW}$ of power is absorbed in the damper for every $3 \mathrm{~kW}$ input power that is supplied. For convenience, two dampers were located in the cavity front covers on either side of the median plane. The damper ceramics were rated and conditioned simultaneously to 1 $\mathrm{kW}$ power absorption at $352 \mathrm{MHz}$.

During the conditioning process, multipactoring was detected on the surface of the damper probe and occurred along the length of the probe that passed through the cavity port, see Fig. 3. This was resolved by coating the probes with approximately $10 \mathrm{~nm}$ of titanium.

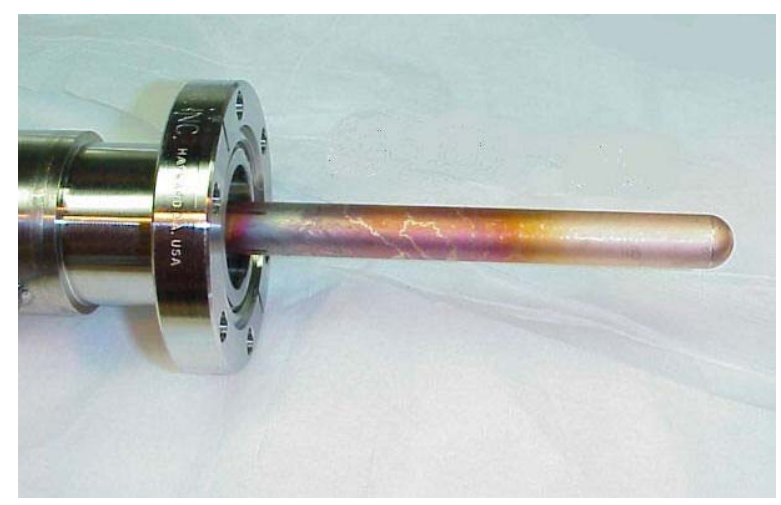

Figure 3: Coaxial damper with probe discoloration due to multipactoring during high-power conditioning.

\section{OPERATIONS}

In order to ensure proper reduction of the HOM Q for 300-mA operation, a factor of eight was determined to be sufficient to deQ the HOM and impose a safety margin beyond the minimum levels reported in [1]. As a result, the dampers were fully inserted into the cavity with the probe extending two inches past the inner wall of the cavity.

The dampers were installed on four cavities in a single sector in the storage ring. Separate flow meters and RTDs were attached to each water circuit for each of the dampers in order to fully monitor the heat load. Cooling water flow rates were set to $1.2 \mathrm{gpm}$ and $0.9 \mathrm{gpm}$ for the ceramic and probe water circuits, respectively. The flow rates, RTD temperature readings, and the calculated power absorption levels were continuously monitored by a Kyoto PLC that was remotely accessed by EPICS [4].
Once installed, the dampers performed as expected. At $100-\mathrm{kW}$ fundamental mode input power, there was no detectable power loss in the damper ceramic. The damper center probe dissipated $240 \mathrm{~W}$, which compares closely with the calculated value from Table 2 .

Since the installation of the dampers, no instabilities resulting from this mode have been found up to $100 \mathrm{~mA}$ with the nominal fill patterns. However, due to the frequency shift of the HOM by the insertion of the damper, the operating conditions that previously produced the instability can not be recreated and directly compared. Many studies have been performed, and it is believed that the inclusion of the dampers has increased the threshold current limit by at least $50 \%$, at which point other HOMs become predominant.

Since the coaxial damper is inherently a broadband device and its power measurements are made using calorimetry, it is difficult to evaluate the frequencies of the modes that are being absorbed as well as their power levels to a precision greater than tens of watts. During typical operation with a 24-bunch fill pattern, the damper ceramic dissipates roughly $100 \mathrm{~W}$ of power. Based on its limitations, it is difficult to use the dampers as a diagnostic tool to evaluate accurately the interaction of the beam with the cavity and determine the spectral amplitudes creating the $100-\mathrm{W}$ heat load. An effort is being made at the present time to create a new HOM eprobe damper with a detachable rf load that is capable of extracting mode spectrum data to address these limitations.

\section{CONCLUSION}

Coaxial e-probe dampers have recently been installed in four cavities in the storage ring of the APS. They are standard coaxial dampers with a ceramic lossy load. They have been extensively modeled and tested in order to ensure their performance and compatibility in the accelerator. Since their installation, their performance has been adequate although their use as a diagnostic tool has been limited due to the nature of the ceramic load. Dampers with increased capabilities are currently being designed as an eventual replacement.

\section{REFERENCES}

[1] L. Emery, "Required Cavity HOM deQing Calculated from Probability Estimates of Coupled Bunch Instabilities in the APS Ring," PAC'93, Washington, DC, May 1993, p. 3360, http://www.jacow.org.

[2] P. Matthews, Y. Kang, and R. Kustom, "Storage Ring Cavity Higher-Order Mode Dampers for the Advanced Photon Source," PAC'95, Dallas, TX, May, 1995, p. 1684, http://www.jacow.org.

[3] Y. Kang, "HOM Damping with Coaxial Dampers in the Storage Ring Cavities of the Advanced Photon Source", EPAC'94, London, June, 1994, p. 2149, http://www.jacow.org.

[4] Experimental Physics and Industrial Control System, http://www.aps.anl.gov/epics/. 